\title{
Fire Sensors Data Acquisition System and its Transmission by Using C8051 MCU
}

\author{
Ramadhani M. Ally ${ }^{1}$, Zhang Jun ${ }^{2}$ \\ ${ }^{1}$ Tianjin University of Technology and Education, 1310\# Dagu South Road, Hexi District, Tianjin 300222, P.R of China \\ ${ }^{2}$ Professor, Tianjin University of Technology and Education, 1310\# Dagu South Road, Hexi District, Tianjin 300222, P.R of China
}

\begin{abstract}
Fire sensors are the automatic components of the fire alarm system which include sensors for light, smoke, temperature, gas, pressure and humidity. In many buildings, fire alarm systems are equipped with wired or wireless sensors .In these systems, there are two data acquisition techniques used to collect information by using sensor detectors, namely; single-sensor detector and mult-sensor detector. Single sensor detector is vulnerable to interferences, such as dust, electromagnetic waves, water vapour, air, light, vibration and other environmental conditions. Then these fire parameters can be transmitted by using various techniques. In this proposed system we used RS232 protocol in Conjunction with C8051F040 Microcontroller to control and facilitate the said system automation. The data are taken and transmitted by cables up to the Microcontroller for transmission and further Processes.
\end{abstract}

Keywords: Fire Sensors; RS232 protocol; MCU

\section{Introduction}

There are various types of fire alarm sensor systems which are used to extinguish fire when such events happen. Despite of having such large number of said systems; most of them have a number of drawbacks such as low speed of operation, false alarm rate, one parameter operation and others. The proposed system will use a number of sensors so as to cutter for various fire parameters. The fire sensors to be used are temperature sensor, smoke sensor, pressure sensor and humidity sensor. Since we took into consideration enough number of fire parameters, it is promising that the issue of speed and false alarm rate will be solved. By using C8051F040 Microcontroller, RS232 protocol system including its cable, signal of fire can be automated and transmitted. The said MCU to be used will have an embedded program in it. The program will control various tasks required to be done by it. Such tasks are like taking fire parameters threshold values, processing and transmit them to the required destination and other tasks. Silicon Laboratories IDE software was used to run program. However, Keil software is also used because Silicon IDE doesn't have compiler but also it can be used for the same purposes. Also Keil softwareis generally used for all types of microcontrollers. We selected C8051F040microcontroller because of its number of advantages such as speed of about $1 \mathrm{Mps}$, the easy way of adding I/O peripheral devices hence increasing its flexibility and therefore enables an 8-bit portfolio of 8-bit devices. The MCU has also backward capabilities, if these capabilities are enabled. However, the C8051F040 MCU architecture originally supports only 256 Special Function Registers (SFRs). This restriction imposes quite a burden on resource-rich devices. To alleviate this limitation, Silicon Labs introduced a paging capability to the
SFR map. This feature supports the use of much larger register spaces. Some of the larger 8-bit devices released by Silicon Labs use up to five pages. Most of these devices have at least two pages where one page is dedicated for debug control logic and cannot be accessed by the program developer. In the past, most of 8-bit embedded programs have been written in assembly language. Later on due to some reasons like readability, maintenance, capability to move programs to other target areas and others, many microcontrollers including 8-bit ones have compilers which are basically c-compilers like IDE, Keil and others. In the proposed system, we explained about sensors-C8051F040 interfacing mechanism, its data transmission and operation sequences. Fire alarm sensor which is the integration of four sensors measuring temperature, pressure, smoke and humidity. The proposed system has several parts in it, namely: temperature, pressure, smokes and humidity as most critical parameters to which the occurrence of fire depends on them. We also showed the analogy to digital converter (ADC) which in practice is inside of C8051F040microcontroller. In addition we showed the block diagram of C8051F040microcontroller

\section{Architecture of the System}

The architecture of the system is as shown in the figure 1 below. The integrated fire alarm sensor has a number of small sensors in it which measure different parameters associated with fire namely temperature, pressure, smoke and humidity. Other components associated to it are analogy to digital converter (ADC), C8051F040 microcontroller and modulator which is connected to the power line carrier. 
International Journal of Science and Research (IJSR)

ISSN (Online): 2319-7064

Index Copernicus Value (2013): 6.14 | Impact Factor (2014): 5.611

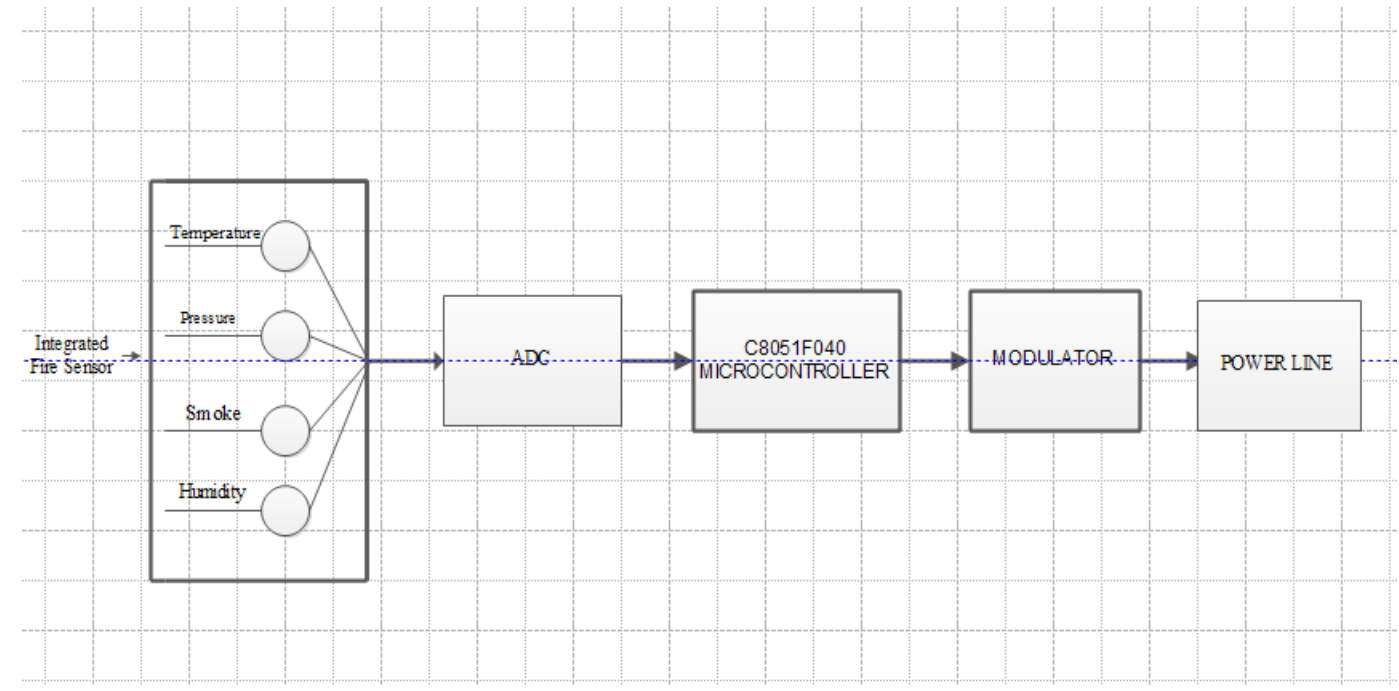

Figure 1: Architecture of the system

When any of the predefined parameters exceeds the threshold value defined in the program, the MCU will detect that value and send the command to one of sensor in the fire alarm integrated system so that it can send that signal to the MCU which will be sent to the transmitter, through the power line carrier up to the receiver point. The function of analog to digital converter (ADC) is to convert any of the said defined parameters from analogy state to digital state so that it can be processed by the MCU. Also the function of MCU in the proposed design is to monitor, translate some signal and control the various tasks required in the operation.

\section{Types of Sensors}

Sensors are selected depending on the applications they are intended for. On that ground there are no direct answers that can be obtained during the selection of sensors for specific applications. In general the following factors can be taken as a guide during the selection of sensors, namely: - (i) suitability in hazardous areas, (ii) cost effectiveness (iii) possibility of being installed at reasonable cost (iv) it should be small so that its installation could be simple (v) it should be safe to use (vi) maintenance and operation should be simple so that it can be monitored even with personnel with little knowledge (vii)it should be reliable (viii)it should have defined working temperature range if it is temperature sensor(ix)the percentage of humidity should be known for humidity sensors.

In the proposed system, four types of sensors were used as explained in the introduction. The first type is the temperature sensor which is commonly known as Resistance Temperature Detector (RTD), which also known as resistance Thermometer. This is the type of sensor which exploits the predictable change in electrical resistance of some materials with change in temperature. The resistance is obtained by measuring constant current and the voltage drop across the resistor. This relationship between voltage and Resistance is well explained by Ohm's law that, voltage is proportional to the Resistance and hence temperature. That means when using RTD's for temperature measurements, the electrical resistance is variable utilized. Currently, the RTD's commonly manufactured by many factories are those with the nominal value of $100 \mathrm{ohms}$ at $0^{\circ} \mathrm{C}$ which is commonly known as Pt-100 resistor and of the value of 1000 ohms at $0{ }^{\circ} \mathrm{C}$ which is also known as Pt-1000. We selected this sensor due to the fact that it can be powered with the same power supply used by MCU because both use the same voltage levels(maximum of 5VDC), can be programmed, doesn't require external components, do not require standby power during idle and waiting time. In the proposed system we used temperature and humidity sensor with model number AM2301 because it can be used to sense both temperature variation as well as humidity as among of the parameters we are using to detect fire. Other advantages of such sensor are accuracy, reliability, stability, fast response, being an 8bit device, ability to resist interference, has calibration chamber for humidity, it use serial interface like RS232, small in size, simple and quicker installation. It has ability to utilize low power, signal transmission distance up to 20 meters.

The sensor can also be used in areas such as weather stations, hospitals, testingof some equipment, home appliances, in motor vehicles and other related areas.

Below is the picture for the digital temperature and humidity sensor which shows the pin connections as well as components associated with it.

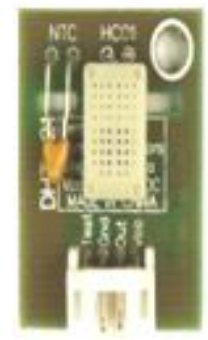

Figure 2

\section{Digital Temperature and Humidity Sensor}

The temperature characteristics for the temperature part can be shown in the figure below which show the variation of voltage and temperature within a given range. 


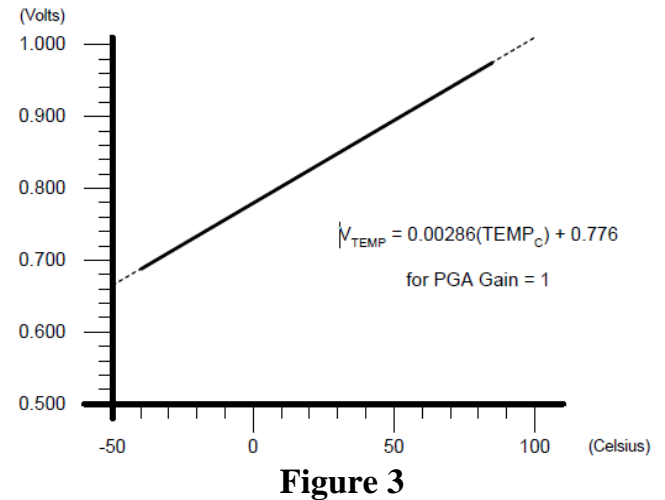

\subsection{Smoke Sensor}

In the proposed system we also have smoke sensor. There are various kinds of smoke sensors in use, some of them are as follows:-

1) Standard smoke sensor: This is a single wave infrared sensor

2) Blue Infrared LED smoke detector: This has a dual wavelength used for commercial applications and has the sensitivity of $5 \% / \mathrm{m}$

3) Blue Infrared smoke detector: This has more rugged version of the blue infrared smoke detector designed for use in aviation areas

4) Hart XL: Is a single wavelength infrared high sensitive smoke detector. This type is mainly for commercial smoke alarms.

5) Very Early Smoke Detector Apparatus (VESDA):Is a single wavelength infra red high sensitivity smoke detector which is similar to Hart XL but it uses filters to remove larger dust particles allowing only smoke fumes through sensitive zones.

\subsection{Pressure Sensor}

The last kind of sensor proposed in this work is the pressure sensor. In principle pressure and temperature are proportional to each other while the volume and other parameters are constant.

The proposed pressure sensor specifications and features are as follows:-

1) Has a pressure of $0.30 \mathrm{bar}$

2) Has 16Bit Analogy to digital converter (ADC)

3) Has 3 wire and is serial interface device

4) Has one system clock line of $32.788 \mathrm{kHz}$

5) Has low voltage as well as low pressure consumption

6) Is a piezo resistive silicon micro machined sensor

7) Has 6 coefficient for soft compensation stored in chip

8) It does not need external component V-T Characteristics

\section{Transmitter Part}

As it can be seen in the figure 4 below, there are a number of blocks namely: - carrier frequency oscillator which is a source of carrier frequency used for signal transmission,C8051F040 microcontroller for controlling inputs, outputs and tasks of the system. Thereafter we have OOK Modulator which is a special case of Amplitude Shift Keying (ASK) whereby no carrier is present during the transmission of a zero. Then we have power amplifier block for signal amplification and thereafter we have interfacing circuit which is used to isolate the $220 \mathrm{~V} / 50 \mathrm{~Hz}$ power line from the low voltage environment.

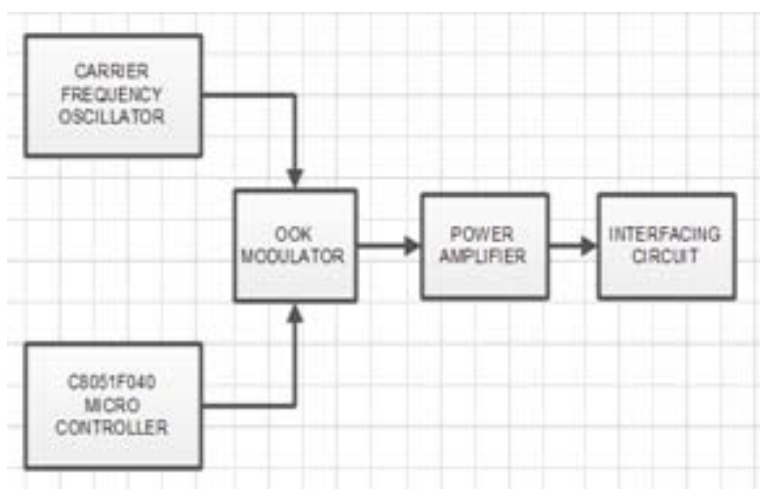

Figure 4: Transmitter Block Diagram

\section{Program Execution}

The execution of the program software is governed by the program flow chart shown in the figure 5below. The first step is to initialize the program. Thereafter, do settings and definitions of some involved parameters. Then some commands which help to send data to the required destination willfollow. After that there will be wait command which will direct the program to wait for some time (say $50 \mu \mathrm{s}$ ). Then the data will be checked to see if it is successful sent, if there are some error the MCU will send again data to the receiver and the process will repeat until the program is stopped.

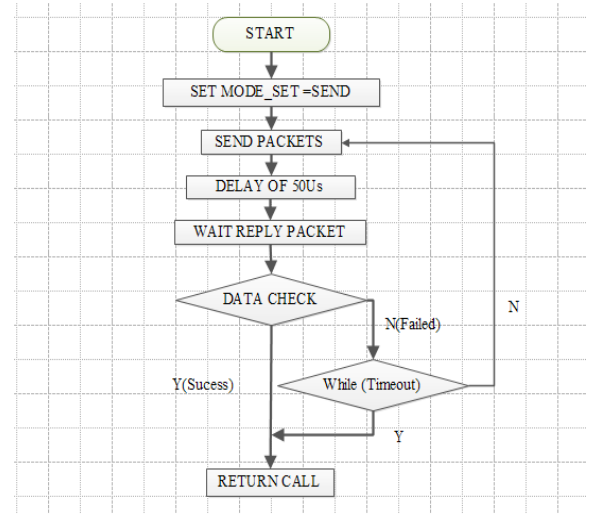

Figure 5: Transmitter Program Flow Chart

\section{Principle of operation}

The circuit uses the Control Area Network (CAN) system in data acquisition as well as data transmission. The information are moved from CAN RAM of C8051F040 microcontroller . The CAN node sensors are configured to take data when the threshold values of the monitored parameters are attained. In fact after threshold values of parameters were attained, the MCU will send command so that the node sensors can start transmitting the data through the C8051F040 MCU up the power line modem. The system is operating at a frequency of $11 \mathrm{MHz}$ as a system clock. The desired Bit rate of $\mathrm{MCU}$ is $1 \mathrm{MBit} / \mathrm{s}$ at bit rate time of 


\section{International Journal of Science and Research (IJSR) \\ ISSN (Online): 2319-7064}

Index Copernicus Value (2013): 6.14 | Impact Factor (2014): 5.611

$1000 \mathrm{~ns}$. System propagation delay time $400 \mathrm{~ns}$ at a bus length of $10 \mathrm{~m}$ with $5 \mathrm{~ns} / \mathrm{m}$ signal delay time.

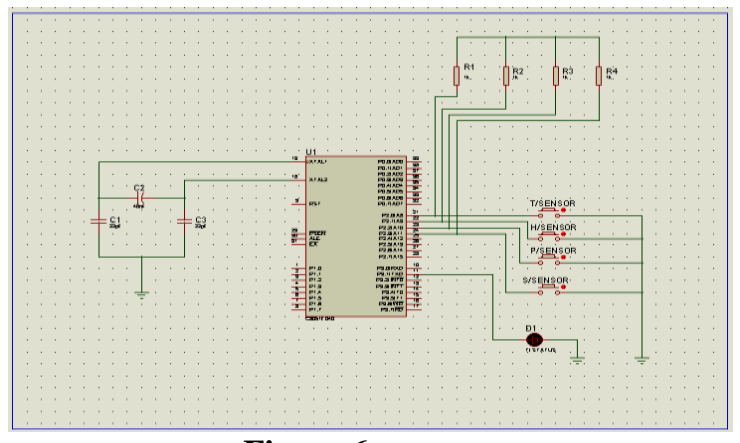

Figure 6

Tra Transmitter Schematic Diagram

\section{Conclusion}

We discussed the designed approach of fire alarm sensor data acquisition and its transmission. The proposed system is designed for low cost residential houses. Thus the system is economical, stable, accurate and fast in response at operating system frequency of $11 \mathrm{MHz}$ and Bit rate of $1 \mathrm{MB} / \mathrm{s}$.

Other applications of the proposed system are short distance data, files and message transmission. It can also be employed in signal and status information display, electrical residential load control such as lights, air conditioners as well as factory machine controls.

\section{References}

[1] Jovita Serrao,Awab Fakih, Ramzan Khatik, Shekh Afazal, Chaya Ravindra, "Transmission of data using power line carrier communication system".

[2] Joseph M.Flemings, "Smoke detectors technology and investigation of fatal fires"

[3] Mode Sollehudin Bin Md Said, "Design of fire alarm system based on power line carrier"

[4] Yute Chen and Jeng-Kuang Hwang,"Powerline based sensor network for pro active electrical fire precaution and early discovery"

[5] Walter W.Yuen,"A Monte carlo approach for the design of thermal fire detection system"

[6] PraveenPatel, "Design of Fire Detection and Alarm System Using PLC SCADA for Shopping Mall"

[7] Meng Xianbo and $\mathrm{Hu}$ xiaoguang,"Design of On-line Temperature Monitoring System for Power Cable Joints Based on Super Capacitor"

[8] Yin Qun and Zhang Jianbo,'Design of Power Line Carrier Communication System Based on FSK-KQ330 Module"

[9] Ashis Garg,"Designing Reliable Power Line Communications"

[10] Inderpreet Kaur."Microcontroller Based Home Automation System",

[11] Suneel Mudunru,"Real Time Security Control System for Smoke and Fire Detection Using Zig Bee" 